\title{
An anisotropic equation of state for high pressure, high temperature applications
}

R. Myhill ${ }^{1}$

This is a non-peer reviewed preprint submitted to EarthArXiv.

\footnotetext{
${ }^{1}$ School of Earth Sciences, University of Bristol.

Wills Memorial Building, Queen's Road, Bristol BS8 1RJ.

bob.myhill@bristol.ac.uk
} 


\title{
An anisotropic equation of state for high pressure, high temperature applications
}

\section{R. Myhill}

Received: date / Accepted: date

\begin{abstract}
This paper presents a strategy for consistently extending isotropic equations of state to model anisotropic materials over a wide range of pressures and temperatures under nearly hydrostatic conditions. The method can be applied to materials of arbitrary symmetry. The paper provides expressions for the deformation gradient tensor, the lattice parameters, the isothermal elastic compliance tensor and thermal expansivity tensor. Scalar properties including the Gibbs energy, volume and heat capacities are inherited from the isotropic equation of state. Other physical properties including the isothermal and isentropic stiffness tensors, the Grüneisen tensor and anisotropic seismic velocities can be derived from these properties.

The equation of state is demonstrated using periclase (cubic) and San Carlos olivine (orthorhombic) as examples.
\end{abstract}

Keywords anisotropic $\cdot$ equation of state $\cdot$ seismic velocities

\section{Introduction}

There exist many treatments of finite elastic deformation in isotropic materials. Early isothermal equations of state were developed by Murnaghan (1937) and Birch (1947), who expanded the Helmholtz free energy as a Taylor expansion in Eulerian finite strain. Extensions to high temperatures have since been derived by Thomsen (1972), Davies (1974) and Stixrude and Lithgow-Bertelloni (2005), who expanded the quasiharmonic vibrational energy to fourth order in finite strain. The final equations assume that isotropic strains induce isotropic stresses, and are therefore valid only for isotropic and cubic media. Co-derived expressions dependent on shear strain (the shear modulus, for

R. Myhill

School of Earth Sciences, University of Bristol

E-mail: bob.myhill@bristol.ac.uk 
example; Stixrude and Lithgow-Bertelloni, 2005) are only valid for isotropic media $^{1}$.

Many alternative approaches to Eulerian finite strain expansions have been published, which rely variously on different measures of strain (e.g. Poirier and Tarantola, 1998), models based on atomic potentials (e.g. Stacey et al, 1981; Vinet et al, 1987) and physical constraints on ultrahigh pressure behaviour (Keane, 1954; Anderson, 1968; Stacey and Davis, 2004). All of these approaches provide scalar properties as a function of pressure/volume and temperature/entropy, and do not consider anisotropy.

A self-consistent anisotropic equation of state is desirable for at least two reasons. Firstly, it would allow lab and computational experimentalists to simultaneously and consistently fit different kinds of data to an anisotropic model, such as cell parameters from X-ray diffraction and seismic velocities from Brillouin spectroscopy (Marquardt and Thomson, 2020) or isothermal compressibilities from ab-initio simulations (Stackhouse and Brodholt, 2007). Secondly, having a compact equation of state would allow geophysicists to directly apply experimentally-derived models to their own field of study.

The development of a practical anisotropic equation of state has been hindered by mismatched "natural" variables. Finite strain is the natural tensor for developing equations of state, because it is intimately connected to lattice elastodynamics (Born and Huang, 1954; Ita and Stixrude, 1992; Stacey and Davis, 2004). Conversely, stress is a natural tensor to use in most real-world applications, because elastic behaviour is typically restricted to conditions of small deviatoric stress. Therefore, while expressing the Helmholtz energy as a Taylor expansion in finite strain makes sense from an atomistic perspective, the expansion order required to cover reasonable ranges of pressure and temperature would result in many hundreds of parameters, covering regions of strain space which could never be accessed by real materials. Analytical expressions for many simple material properties (such as the volume) would simply be too unwieldy to be useful.

This paper outlines an alternative method of constructing an anisotropic equation of state using an isotropic equation of state as a basis. This method offers great potential, because isotropic equations of state provide a natural strain measure $\left(\ln \left(V / V_{0}\right)\right)$ that can be used in expansions of elastic properties. Section 2 describes the generalisation of the isotropic thermodynamic relations to anisotropic materials. Section 3 derives an equation for the finite deformation gradient tensor. Section 4 describes the formulation for the anisotropic equation of state. Section 5 derives the key formulae to enable calculation of thermodynamic/thermoelastic properties as a function of pressure and tem-

1 The shear moduli of cubic materials have distinct Voigt and Reuss bounds, with the difference given by the following expression:

$$
G_{V}-G_{R}=\frac{3}{5} \frac{\left(C_{11}-C_{12}-2 C_{44}\right)^{2}}{\left(3 C_{11}-3 C_{12}+4 C_{44}\right)}
$$

. The bulk moduli of cubic materials have identical Voigt and Reuss bounds. 
perature. Section 6 outlines the algorithm for practical use of the equation of state. Finally, examples of the new equation of state are given in Section 7.

Symbols used in this paper are presented in Table 1.

Table 1 Symbols used in this paper

\begin{tabular}{|c|c|c|}
\hline Symbol & Units & Description \\
\hline $\mathcal{E}$ & $\mathrm{J} \mathrm{mol}^{-1}$ & Molar Internal energy \\
\hline $\mathcal{F}$ & $\mathrm{J} \mathrm{mol}^{-1}$ & Molar Helmholtz energy \\
\hline $\mathcal{G}$ & $\mathrm{J} \mathrm{mol}^{-1}$ & Molar Gibbs energy \\
\hline $\boldsymbol{x}, x_{i}$ & $\mathrm{~m}$ & Position in spatial reference frame \\
\hline $\boldsymbol{F}, F_{i j}$ & [unitless] & Deformation gradient tensor, $\left(\partial \boldsymbol{x} / \partial \boldsymbol{x}_{0}\right)$ \\
\hline $\boldsymbol{\sigma}, \sigma_{i j}$ & $\mathrm{~Pa}$ & Cauchy stress (or true stress) \\
\hline$T$ & K & Temperature \\
\hline$e, e_{i j}$ & [unitless] & Finite strain measure work-conjugate to $\sigma$ \\
\hline$\varepsilon, \varepsilon_{i j}$ & [unitless] & Infinitesimal strain tensor \\
\hline$N$ & $\mathrm{~J} \mathrm{~K}^{-1} \mathrm{~mol}^{-1}$ & Molar entropy \\
\hline$V$ & $\mathrm{~m}^{3} \mathrm{~mol}^{-1}$ & Molar volume \\
\hline$P$ & $\mathrm{~Pa}$ & Pressure $\left(\delta_{i j} \sigma_{i j} / 3\right)$ \\
\hline$s, s_{i j}$ & $\mathrm{~Pa}$ & Deviatoric stress tensor $\left(\sigma_{i j}-\delta_{i j} P\right)$ \\
\hline $\mathbb{S}_{\mathrm{T}}, S_{\mathrm{T} i j k l}, S_{\mathrm{T} p q}$ & $\mathrm{~Pa}^{-1}$ & Isothermal compliance tensor (standard and Voigt form) \\
\hline $\mathbb{S}_{\mathrm{N}}, S_{\mathrm{N} i j k l}, S_{\mathrm{N} p q}$ & $\mathrm{~Pa}^{-1}$ & Isentropic compliance tensor (standard and Voigt form) \\
\hline $\mathbb{C}_{\mathrm{T}}, C_{\mathrm{T} i j k l}, C_{\mathrm{T} p q}$ & $\mathrm{~Pa}$ & Isothermal stiffness tensor (standard and Voigt form) \\
\hline $\mathbb{C}_{\mathrm{N}}, C_{\mathrm{N} i j k l}, C_{\mathrm{N} p q}$ & $\mathrm{~Pa}$ & Isentropic stiffness tensor (standard and Voigt form) \\
\hline$\alpha, \alpha_{i j}$ & $\mathrm{~K}^{-1}$ & Thermal expansivity tensor \\
\hline$\alpha_{V}$ & $\mathrm{~K}^{-1}$ & Volumetric thermal expansivity \\
\hline$C_{\sigma}$ & $\mathrm{J} \mathrm{K}^{-1} \mathrm{~mol}^{-1}$ & Molar isostress heat capacity \\
\hline$C_{V}$ & $\mathrm{~J} \mathrm{~K}^{-1} \mathrm{~mol}^{-1}$ & Molar isochoric heat capacity \\
\hline$C_{P}$ & $\mathrm{~J} \mathrm{~K}^{-1} \mathrm{~mol}^{-1}$ & Molar isobaric heat capacity \\
\hline$\beta_{T R}$ & $\mathrm{~Pa}^{-1}$ & Isothermal Reuss compressibility \\
\hline$K_{T R}$ & $\mathrm{~Pa}^{-1}$ & Isothermal Reuss bulk modulus \\
\hline$\rho$ & $\mathrm{kg} \mathrm{m}^{-3}$ & Density \\
\hline$\gamma, \gamma_{i j}$ & [unitless] & Grüneisen tensor \\
\hline$P_{\text {th }}$ & $\mathrm{Pa}$ & Thermal pressure \\
\hline$f$ & [unitless] & Logarithmic volume change $\left(\ln \left(V / V_{0}\right)\right)$ \\
\hline$\chi_{i j k l}, \chi_{p q}$ & [unitless] & Anisotropic state function \\
\hline$c_{i j k l m n}, c_{p q m n}$ & $\mathrm{~Pa}^{-\mathrm{n}}$ & Constants defining $\chi_{i j k l}$ as a function of $f$ and $P_{\mathrm{th}}$ \\
\hline$M, M_{i j}$ & & Deformed unit vector matrix \\
\hline$Q, Q_{i j}$ & [unitless] & Coordinate system transformation matrix \\
\hline $\boldsymbol{R}, R_{i j}$ & [unitless] & Material rotation matrix \\
\hline $\boldsymbol{I}, \delta_{i j}$ & & Identity matrix / Kronecker delta \\
\hline $\ln _{\mathrm{M}}()$ & & Matrix logarithm function \\
\hline $\exp _{M}()$ & & Matrix exponential function \\
\hline
\end{tabular}

\section{Material properties as functions of stress and temperature}

Thermodynamic and thermoelastic properties of materials under given stress and temperature conditions are related to the Gibbs energy and its derivatives. For the purposes of this study, we express the molar Gibbs energy as $\mathcal{G}(\boldsymbol{\sigma}, T)$, where $\boldsymbol{\sigma}$ is the Cauchy stress (also known as the true stress). The first derivatives of $\mathcal{G}$ are related to a finite strain measure (which will not be 
elaborated on in this study), molar volume $V$ and molar entropy $N$ :

$$
\begin{aligned}
e & =-\left.\frac{1}{V} \frac{\partial \mathcal{G}}{\partial \boldsymbol{\sigma}}\right|_{T} \\
N & =-\left.\frac{\partial \mathcal{G}}{\partial T}\right|_{\boldsymbol{\sigma}}
\end{aligned}
$$

The pressure derivative of the molar Gibbs energy is the molar volume:

$$
V=\left.\frac{\partial \mathcal{G}}{\partial P}\right|_{T}
$$

Second derivatives of $\mathcal{G}$ with respect to $\sigma$ and $T$ yield the fourth rank isothermal compliance tensor $\mathbb{S}_{\mathrm{T}}$, second rank thermal expansivity tensor $\boldsymbol{\alpha}$ and (scalar) molar isostress heat capacity $C_{\boldsymbol{\sigma}}$ :

$$
\begin{aligned}
\mathbb{S}_{\mathrm{T}} & =\left.\frac{\partial \boldsymbol{\varepsilon}}{\partial \boldsymbol{\sigma}}\right|_{T}=-\left.\frac{1}{V} \frac{\partial^{2} \mathcal{G}}{\partial \boldsymbol{\sigma} \partial \boldsymbol{\sigma}}\right|_{T} \\
\boldsymbol{\alpha} & =\frac{1}{V} \frac{\partial^{2} \mathcal{G}}{\partial \boldsymbol{\sigma} \partial T} \\
C_{\boldsymbol{\sigma}} & =-\left.T \frac{\partial^{2} \mathcal{G}}{\partial T^{2}}\right|_{\boldsymbol{\sigma}}
\end{aligned}
$$

where $\varepsilon$ is the infinitesimal strain tensor. Under hydrostatic conditions, $P=$ $\delta_{i j} \sigma_{i j}$, leading to a scalar measure for the compliance tensor:

$$
\begin{aligned}
\left.\left.S_{\mathrm{T} i j k l} \frac{\partial \sigma_{i j}}{\partial P}\right|_{T} \frac{\partial \sigma_{k l}}{\partial P}\right|_{T} & =-\left.\left.\left.\frac{1}{V} \frac{\partial^{2} \mathcal{G}}{\partial \sigma_{i j} \partial \sigma_{k l}}\right|_{T} \frac{\partial \sigma_{i j}}{\partial P}\right|_{T} \frac{\partial \sigma_{k l}}{\partial P}\right|_{T} \\
S_{\mathrm{T} i j k l} \delta_{i j} \delta_{k l} & =-\left.\frac{1}{V} \frac{\partial^{2} \mathcal{G}}{\partial P \partial P}\right|_{T} \\
& =-\left.\frac{1}{V} \frac{\partial V}{\partial P}\right|_{T} \\
& =-\left.\frac{\partial \ln V}{\partial P}\right|_{T}
\end{aligned}
$$

This is the definition of the isothermal Reuss (isostress) compressibility $\beta_{\mathrm{TR}}$ (the inverse of the isothermal Reuss bulk modulus $K_{\mathrm{TR}}$ ):

$$
\beta_{\mathrm{TR}}=\frac{1}{K_{\mathrm{TR}}}=S_{\mathrm{T} i j k l} \delta_{i j} \delta_{k l}
$$

The volume can therefore be expressed as a function of the compressibility:

$$
\frac{V}{V_{0}}=\exp \left(\int_{0}^{P}-\beta_{\mathrm{TR}} d P\right)
$$


The isentropic compliance tensor $\mathbb{S}_{N}$, molar isochoric heat capacity $C_{V}$, volumetric thermal expansivity $\alpha_{V}$ and density of media subjected to hydrostatic stresses are as follows (Nye et al, 1985, p178):

$$
\begin{aligned}
\mathbb{S}_{N} & =\mathbb{S}_{\mathrm{T}}-\boldsymbol{\alpha} \boldsymbol{\alpha} \frac{V T}{C_{P}} \\
C_{V} & =C_{P}-\frac{\alpha_{V}^{2} V T}{\beta_{\mathrm{TR}}} \\
\alpha_{V} & =\operatorname{tr}(\boldsymbol{\alpha}) \\
\rho & =\frac{M}{V}
\end{aligned}
$$

where $M$ is the molar mass and we have equated the molar isostress heat capacity $C_{\boldsymbol{\sigma}}$ with the molar isobaric heat capacity $C_{P}$. Finally, the Grüneisen tensor may be calculated (Appendix A) using the isentropic elastic stiffness tensor $\mathbb{C}_{\mathrm{N}}=\left(\mathbb{S}_{\mathrm{N}}\right)^{-1}$ :

$$
\gamma=\mathbb{C}_{\mathrm{N}} \boldsymbol{\alpha} \frac{V}{C_{\boldsymbol{\sigma}}}
$$

\section{The finite deformation gradient tensor}

In order to describe pressure and temperature-induced changes in the unit cell lattice, we must define the deformation gradient tensor $\boldsymbol{F}$ as a function of the compliance tensor. The deformation gradient tensor describes the change in position of an element of the deformed crystal relative to that in the undeformed reference state:

$$
F_{i j}=\frac{\partial x_{i}}{\partial x_{j 0}}
$$

Any position $\boldsymbol{x}_{0}$ in the undeformed crystal will move to position $\boldsymbol{x}$ after a homogeneous deformation, where

$$
x_{i}=F_{i j} x_{j 0}
$$

In the limit of small deformation, the deformation gradient tensor is related to the infinitesimal strain tensor:

$$
\begin{aligned}
F_{i j} & =\frac{\partial x_{i 0}}{\partial x_{j 0}}+\frac{\partial\left(x_{i}-x_{i 0}\right)}{\partial x_{j 0}} \\
& =\delta_{i j}+\varepsilon_{i j}
\end{aligned}
$$

Let us now consider the deformation of an anisotropic crystal along a finite isothermal pressure path. For each infinitesimal pressure step $\delta P$, the deformation gradient tensor from the previous step $\boldsymbol{F}(P)$ is pre-multiplied by a nearly diagonal matrix:

$$
F_{i k}(P+\delta P)=\left(\delta_{i j}+\varepsilon_{i j}\right) F_{j k}(P)
$$


It is useful here to take the matrix logarithm of both sides of the equation:

$$
\ln _{\mathrm{M}}(\boldsymbol{F}(P+\delta P))=\ln _{\mathrm{M}}((\boldsymbol{I}+\boldsymbol{\varepsilon}) \boldsymbol{F}(P))
$$

The two matrices commute for small $\varepsilon$, allowing the separation into two matrix logarithms:

$$
\ln _{\mathrm{M}}(\boldsymbol{F}(P+\delta P))=\ln _{\mathrm{M}}(\boldsymbol{F}(P))+\ln _{\mathrm{M}}(\boldsymbol{I}+\boldsymbol{\varepsilon})
$$

Making use of Equation 5 and applying the limit

$$
\lim _{\boldsymbol{\varepsilon} \rightarrow \mathbf{0}} \ln _{\mathrm{M}}(\boldsymbol{\varepsilon}+\boldsymbol{I})=\varepsilon
$$

allows a small change in $\ln _{\mathrm{M}} \boldsymbol{F}$ to be expressed as a function of $\delta P$ :

$$
\begin{aligned}
\ln _{\mathrm{M}}(\boldsymbol{F}(P+\delta P)) & =\ln _{\mathrm{M}}(\boldsymbol{F}(P))+\ln _{\mathrm{M}}\left(\boldsymbol{I}-\mathbb{S}_{\mathrm{T}} \boldsymbol{I} \delta P\right) \\
& =\ln _{\mathrm{M}}(\boldsymbol{F}(P))-\mathbb{S}_{\mathrm{T}} \boldsymbol{I} \delta P \\
\delta\left(\ln _{\mathrm{M}}(\boldsymbol{F})\right) & =-\mathbb{S}_{\mathrm{T}} \boldsymbol{I} \delta P
\end{aligned}
$$

such that

$$
\left.\frac{\partial\left(\ln _{\mathrm{M}} \boldsymbol{F}\right)_{i j}}{\partial P}\right|_{T}=-S_{\mathrm{T} i j k l} \delta_{k l}
$$

Here, $\mathbb{S}_{T}$ is the isothermal compliance tensor in the spatial reference frame, rather than the material reference frame. This has particular implications for monoclinic and triclinic materials, where the material reference frame rotates relative to the spatial reference frame during deformation (see Section 5.3). The deformation gradient tensor relating the undeformed state to a deformed state at pressure $P$ can then be calculated by matrix exponentiation $\left(\exp _{M}\right)$ :

$$
F_{i j}=\frac{x_{i}}{x_{j 0}}=\exp _{\mathrm{M}}\left(\int_{P 0}^{P}-S_{\text {Tijkl }} \delta_{k l} d P\right)
$$

\section{An anisotropic equation of state}

4.1 Reference isotropic equation of state

The anisotropic equation of state described in the following section assumes that the volume (or rather $f=\ln \left(V / V_{0}\right)$ ) and thermal pressure

$$
P_{\text {th }}(f, T)=P(f, T)-P\left(f, T_{\text {ref }}\right)
$$

can be pre-calculated at any desired pressure and temperature. These two properties are provided by isotropic equations of state, of which there are several that faithfully represent volume as a function of pressure and temperature to extreme conditions. Two commonly using equations of state for the deep Earth are those of Stixrude and Lithgow-Bertelloni (2005) and Holland and Powell (2011) (used as a basis for the modelsets of Stixrude and LithgowBertelloni (2011) and Holland et al (2013) respectively). The anisotropic equation of state presented here is agnostic to the choice of basis; the user may choose the best reference equation of state for the material of interest. 
4.2 Extension to anisotropic materials

The relationship between the deformation gradient tensor and isothermal compliance tensor (Equation 30) suggests that it would be convenient to formulate anisotropic equations of state in terms of a tensor state function $\chi_{i j k l}$, where:

$$
\left.\frac{\partial \chi_{i j k l}}{\partial P}\right|_{T}=-S_{\mathrm{T} i j k l}
$$

Both $\chi_{i j k l}$ and $S_{\text {Tijkl }}$ are defined in the spatial reference frame. This allows the deformation gradient tensor (Equation 31) to be calculated directly:

$$
F_{i j}=\exp _{\mathrm{M}}\left(\chi_{i j k l} \delta_{k l}\right)
$$

We now choose a form for the tensor function $\chi_{i j k l}$. One of the most important constraints is that given by Equation 13, i.e.:

$$
\begin{aligned}
f & =\ln \left(\frac{V}{V_{0}}\right) \\
& =\int_{0}^{P}-S_{\mathrm{T} i j k l} \delta_{i j} \delta_{k l} d P \\
& =\left(\chi_{i j k l}-\chi_{i j k l 0}\right) \delta_{i j} \delta_{k l} \\
& =\chi_{i j k l} \delta_{i j} \delta_{k l}
\end{aligned}
$$

where in the last expression we have dropped the arbitrary constant of integration. This constraint can easily be satisfied (Section 4.3) if the isothermal part of $\chi_{i j k l}$ is expressed in powers of $f$ :

$$
\begin{aligned}
\left.\frac{\partial \chi_{i j k l}}{\partial f}\right|_{P_{\mathrm{th}}=0} & =\frac{S_{\mathrm{T} i j k l}}{\beta_{\mathrm{RT}}} \\
& =c_{i j k l 10}+c_{i j k l 20} f+c_{i j k l 30} f^{2}+\ldots \\
\chi_{i j k l}\left(f, P_{\mathrm{th}}=0\right) & =c_{i j k l 10} f+c_{i j k l 20} \frac{f^{2}}{2}+c_{i j k l 30} \frac{f^{3}}{3}+\ldots
\end{aligned}
$$

Extending these expressions to temperatures other than the reference temperature, we must consider that $\partial \mathbb{S} /\left.\partial T\right|_{V} \rightarrow \mathbf{0}$ as $T \rightarrow 0$. This constraint is naturally satisfied if we expand $\chi_{i j k l}$ in powers of $P_{\mathrm{th}}$, because $\partial P_{\mathrm{th}} /\left.\partial T\right|_{V} \rightarrow \mathbf{0}$ 
as $T \rightarrow 0$. Thus, we define $\chi_{i j k l}$ and its partial derivatives as follows:

$$
\begin{aligned}
\chi_{i j k l} & =\chi_{i j k l}\left(f, P_{\mathrm{th}}\right) \\
& =\sum_{n=0}^{n_{n}}\left(\begin{array}{c}
c_{i j k l 0 n}+c_{i j k l 1 n} f+c_{i j k l 2 n} \frac{f^{2}}{2} \\
+c_{i j k l 3 n} \frac{f^{3}}{3}+\ldots
\end{array}\right) P_{\mathrm{th}}^{n} \\
\left.\frac{\partial \chi_{i j k l}}{\partial P_{\mathrm{th}}}\right|_{f} & =\sum_{n=1}^{n_{n}}\left(\begin{array}{c}
c_{i j k l 0 n}+c_{i j k l 1 n} f+c_{i j k l 2 n} \frac{f^{2}}{2} \\
+c_{i j k l 3 n} \frac{f^{3}}{3}+\ldots
\end{array}\right) n P_{\mathrm{th}}^{n-1} \\
\left.\frac{\partial \chi_{i j k l}}{\partial f}\right|_{P_{\mathrm{th}}} & =\sum_{n=0}^{n_{n}}\left(c_{i j k l 1 n}+c_{i j k l 2 n} f+c_{i j k l 3 n} f^{2}+\ldots\right) P_{\mathrm{th}}^{n}
\end{aligned}
$$

The $c_{i j k l m n}$ parameters represent empirical constants, and $c_{i j k l 00}$ parameters are set equal to zero. Symmetry requirements mean that many of these parameters will be set equal to zero, and many more will be set equal to each other (Section 4.3). Low order expansions in $m$ and $n$ are sufficient to reproduce experimental results to a high degree of accuracy (see Section 7). Indeed, Thurston (1967) showed that just three non-zero parameters (constant $\sum_{k} c_{i i k k 10}$ for $1 \leq i \leq 3$, all other parameters equal to zero) were able to accurately reproduce room temperature lattice parameters to $>10 \mathrm{GPa}$ in quartz, magnesium and cadmium.

\subsection{Consistency constraints}

There are several constraints on the forms of the $c_{i j k l m n}$ parameters. Firstly, the values of $c_{i j k l m n}$ must obey the same symmetry and parameter relationships as the underlying compliance tensor $S_{i j k l}$. It is easiest to discuss this in terms of the structure in Voigt notation, contracting the $3 \times 3 \times 3 \times 3$ compliance tensor into a $6 \mathrm{x} 6$ matrix. This study uses the original convention of Voigt (1928) (also used by Mainprice et al, 2011):

$$
\sigma_{p}=C_{\mathrm{T} p q} \eta_{q}
$$

where each element of $C_{\mathrm{T} p q}$ corresponds to an element of the original $C_{\mathrm{T} i j k l}$ tensor. The mapping between each Voigt index $p$ or $q$ and the raw indices $i j, j i$ or $k l, k l$ is given by:

$$
i j \rightarrow p, k l \rightarrow q ; 11 \rightarrow 1 ; 22 \rightarrow 2 ; 33 \rightarrow 3 ; 23 \rightarrow 4 ; 13 \rightarrow 5 ; 12 \rightarrow 6
$$

The elements of the strain vector are $\eta_{1,2,3}=\varepsilon_{11,22,33}$ and $\eta_{4,5,6}=2 \varepsilon_{23,13,12}$. The factor of two is required to preserve the original relationship between stress and strain. The conversion of each element of the compliance tensor 
from the original fourth-rank tensor involves the following factors (Mainprice et al, 2011):

$$
\begin{aligned}
S_{\mathrm{T} p q}= & P S_{\mathrm{T} i j k l} \\
P & = \begin{cases}1 & \text { if } p \in\{1,2,3\} \text { and } q \in\{1,2,3\} \\
2 & \text { if } p \in\{4,5,6\} \text { or } q \in\{4,5,6\} \\
4 & \text { if } p \in\{4,5,6\} \text { and } q \in\{4,5,6\}\end{cases}
\end{aligned}
$$

Contracting $c_{i j k l m n}$ into Voigt form $\left(c_{p q m n}\right)$ yields the following relationships for cubic symmetry:

$$
\begin{aligned}
& -c_{11 m n}=c_{22 m n}=c_{33 m n} \\
& -c_{12 m n}=c_{13 m n}=c_{21 m n}=c_{23 m n}=c_{31 m n}=c_{32 m n} \\
& -c_{44 m n}=c_{55 m n}=c_{66 m n}, \\
& -c_{p q m n}=0 \text { for } p>3 \text { and } / \text { or } q>3 \text { and } p \neq q
\end{aligned}
$$

Linear dependencies should also be preserved. For the hexagonal and rhombohedral classes the parameters should satisfy the following equations: $c_{66 m n}=$ $2\left(c_{11 m n}-c_{12 m n}\right)$. For the rhombohedral classes, the parameters should satisfy these equations: $c_{24 m n}=-c_{14 m n}, c_{25 m n}=-c_{15 m n}, c_{46 m n}=-2 c_{15 m n}$ and $c_{56 m n}=2 c_{14 m n}$.

The volumetric equality $f=\chi_{i j k l} \delta_{i j} \delta_{k l}$ (Equation 38) should also be satisfied. In Voigt form, this is guaranteed if:

$$
\sum_{p=1}^{3} \sum_{q=1}^{3} c_{p q m n}= \begin{cases}1 & \text { if } m=1 \text { and } n=0 \\ 0 & \text { otherwise }\end{cases}
$$

\section{Thermodynamic properties}

\section{1 "Isotropic" properties}

Under hydrostatic stresses, the scalar properties returned by the reference isotropic equation of state are consistent with the anisotropic equation of state. These include the molar Gibbs energy $\mathcal{G}$, the molar entropy $N$, the volume $V$ and all higher derivatives including the volumetric thermal expansivity, isothermal and isentropic Reuss compressibilities and isothermal and isentropic heat capacities.

\subsection{Anisotropic properties}

Anisotropic properties are calculated using the expressions for $\chi_{i j k l}$ and its partial derivatives (Equations 43-45), supplemented by information from the isotropic equation of state. The deformation gradient tensor is given by the expression:

$$
F_{i j}=\exp _{\mathrm{M}}\left(\chi_{i j k l} \delta_{k l}\right)
$$


The isothermal compliance and thermal expansivity tensors are derived by first writing out the total derivative of $\chi_{i j k l}$ with respect to $P$ and $T$ via repeated use of the chain rule:

$$
\begin{aligned}
d \chi_{i j k l} & =d \chi_{i j k l}\left(f(P, T), P_{\mathrm{th}}(f(P, T), T)\right) \\
& =g_{i j k l} d P+h_{i j k l} d T
\end{aligned}
$$

where $g_{i j k l}$ and $h_{i j k l}$ are functions:

$$
\begin{aligned}
g_{i j k l} & =\left.\left.\frac{\partial \chi_{i j k l}}{\partial f}\right|_{P_{\mathrm{th}}} \frac{\partial f}{\partial P}\right|_{T}+\left.\left.\left.\frac{\partial \chi_{i j k l}}{\partial P_{\mathrm{th}}}\right|_{f} \frac{\partial P_{\mathrm{th}}}{\partial f}\right|_{T} \frac{\partial f}{\partial P}\right|_{T} \\
& =-\beta_{\mathrm{RT}}\left(\left.\frac{\partial \chi_{i j k l}}{\partial f}\right|_{P_{\mathrm{th}}}+\left.\left.\frac{\partial \chi_{i j k l}}{\partial P_{\mathrm{th}}}\right|_{f} \frac{\partial P_{\mathrm{th}}}{\partial f}\right|_{T}\right) \\
h_{i j k l} & =\left.\left.\frac{\partial \chi_{i j k l}}{\partial f}\right|_{P_{\mathrm{th}}} \frac{\partial f}{\partial T}\right|_{P}+\left.\left.\left.\frac{\partial \chi_{i j k l}}{\partial P_{\mathrm{th}}}\right|_{f} \frac{\partial P_{\mathrm{th}}}{\partial f}\right|_{T} \frac{\partial f}{\partial T}\right|_{P}+\left.\left.\frac{\partial \chi_{i j k l}}{\partial P_{\mathrm{th}}}\right|_{f} \frac{\partial P_{\mathrm{th}}}{\partial T}\right|_{f} \\
& =\alpha_{V}\left(\left.\frac{\partial \chi_{i j k l}}{\partial f}\right|_{P_{\mathrm{th}}}+\left.\left.\frac{\partial \chi_{i j k l}}{\partial P_{\mathrm{th}}}\right|_{f} \frac{\partial P_{\mathrm{th}}}{\partial f}\right|_{T}\right)+\left.\left.\frac{\partial \chi_{i j k l}}{\partial P_{\mathrm{th}}}\right|_{f} \frac{\partial P}{\partial T}\right|_{V} \\
& =\alpha_{V}\left(\left.\frac{\partial \chi_{i j k l}}{\partial f}\right|_{P_{\mathrm{th}}}+\left.\left.\frac{\partial \chi_{i j k l}}{\partial P_{\mathrm{th}}}\right|_{f} \frac{\partial P_{\mathrm{th}}}{\partial f}\right|_{T}\right)+\left.\frac{\partial \chi_{i j k l}}{\partial P_{\mathrm{th}}}\right|_{f} \frac{\alpha_{V}}{\beta_{\mathrm{RT}}}
\end{aligned}
$$

Taking the partial derivative of Equation 53 with respect to pressure, and using the definition of $\chi$ (Equation 33), we obtain the isothermal compliance tensor:

$$
\begin{aligned}
S_{\mathrm{T} i j k l} & =-\left.\frac{\partial \chi_{i j k l}}{\partial P}\right|_{T} \\
& =\beta_{\mathrm{RT}}\left(\left.\frac{\partial \chi_{i j k l}}{\partial f}\right|_{P_{\mathrm{th}}}+\left.\left.\frac{\partial \chi_{i j k l}}{\partial P_{\mathrm{th}}}\right|_{f} \frac{\partial P_{\mathrm{th}}}{\partial f}\right|_{T}\right)
\end{aligned}
$$

The thermal expansivity tensor can be derived by exploiting the identity:

$$
\begin{gathered}
\frac{\partial}{\partial \boldsymbol{\sigma}}\left(\frac{\partial^{2} \mathcal{G}}{\partial \boldsymbol{\sigma} \partial T}\right)=\frac{\partial}{\partial T}\left(\frac{\partial^{2} \mathcal{G}}{\partial \boldsymbol{\sigma} \partial \boldsymbol{\sigma}}\right) \\
\alpha_{i j}=\frac{1}{V} \frac{\partial^{2} \mathcal{G}}{\partial \sigma_{i j} \partial T} \\
=-\frac{\partial}{\partial T}\left(\int S_{\mathrm{T} i j k l} \delta_{k l} d P\right)_{P} \\
=\left.\frac{\partial \chi_{i j k l}}{\partial T}\right|_{P} \delta_{k l} \\
=\alpha_{V}\left(\left.\frac{\partial \chi_{i j k l}}{\partial f}\right|_{P_{\mathrm{th}}}+\left.\frac{\partial \chi_{i j k l}}{\partial P_{\mathrm{th}}}\right|_{f}\left(\left.\frac{\partial P_{\mathrm{th}}}{\partial f}\right|_{T}+\frac{1}{\beta_{\mathrm{RT}}}\right)\right) \delta_{k l}
\end{gathered}
$$


Other properties can be calculated using the relations in Section 2. These include the isentropic compliance tensor $\left(\mathbb{S}_{N}\right.$; Equation 14) and the Grüneisen tensor ( $\boldsymbol{\gamma}$; Equation 18). The isothermal and isentropic stiffness tensors can be calculated by inverting the corresponding compliance tensors:

$$
\begin{aligned}
\mathbb{C}_{T} & =\left(\mathbb{S}_{T}\right)^{-1} \\
\mathbb{C}_{N} & =\left(\mathbb{S}_{N}\right)^{-1}
\end{aligned}
$$

Once the isentropic compliance and stiffness tensors have been calculated, seismic velocities and associated properties can be calculated. Expressions for these properties are published elsewhere (Mainprice et al, 2011; de Jong et al, 2015).

\subsection{Monoclinic and triclinic systems}

For systems of orthorhombic and higher symmetry, hydrostatic stresses result in diagonal deformation gradient tensors, because the compliance tensor satisfies the following three constraints:

$$
\sum_{q=1}^{3} S_{p q}=0 \text { for } p>3
$$

In these systems, the three sums $\varepsilon_{\mathrm{H} i}=\sum_{k} \chi_{i i k k}$ terms are equal to the Hencky strains along the coordinate axes (Truesdell, 1952; Reiner, 1958).

In monoclinic systems, only two of these constraints are satisfied, and for triclinic systems none are satisfied. This means that any initial alignment between crystallographic and spatial coordinate axes will be lost with changes in pressure and temperature. A rigid body rotation is required to realign the symmetry axes with the spatial reference frame (Figure 1).

In high pressure experiments, elastic tensors are reported relative to the spatial reference frame, but at each individual $P$ - $T$ condition, the crystallographic axes are aligned with that reference frame. In other words, the crystal is rotated to align with the coordinate axes for every measurement. In contrast, the deformation gradient tensor in Section 3 is defined relative to a fixed spatial reference frame. Therefore, for monoclinic or triclinic crystals, the tensor properties must be rotated into the local material reference frame. To define the required rotation, let us define the undeformed unit cell by a set of vector lengths and angles $(a, b, c, \alpha, \beta$ and $\gamma)$, and choose the following convention for the unit vector matrix $\boldsymbol{M}$ which relates the unit cell vectors to the coordinate axes of the spatial reference frame:

$$
\begin{aligned}
\boldsymbol{M}_{0} & =\left[\begin{array}{ccc}
a & 0 & 0 \\
b \cos (\gamma) & b \sin (\gamma) & 0 \\
c \cos (\beta) & c n_{2} & c \sqrt{\sin ^{2}(\beta)-n_{2}^{2}}
\end{array}\right] \\
n_{2} & =\frac{\cos (\alpha)-\cos (\gamma) \cos (\beta)}{\sin (\gamma)}
\end{aligned}
$$




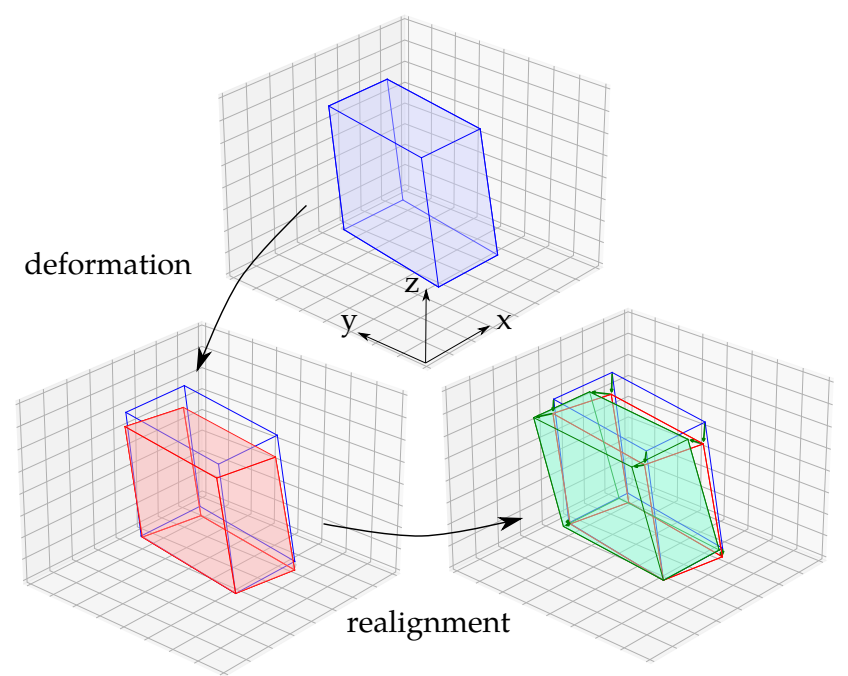

Fig. 1 Deformation and rotation of a triclinic material. In the undeformed reference state, the crystal $a$-axis is colinear with the coordinate $x$-axis, and the crystal $b$-axis is perpendicular to the $z$-axis (top diagram). During hydrostatic deformation, the relationship between crystal cell and coordinate axes is lost (bottom left). A rotation is required to reattain the relationship with the symmetry axes (bottom right). This realignment is only necessary for monoclinic and triclinic systems; in orthotropic systems, relationships between material and spatial coordinate systems are maintained during hydrostatic compression and thermal expansion.

In this convention, the first spatial coordinate axis is colinear with $M_{1}$, the third is orthogonal to $M_{1}$ and $M_{2}$ and the second is orthogonal to the other two axes such that the coordinate system is right-handed.

After deformation, the new unit cell vectors $\boldsymbol{M}$ can be calculated using the deformation gradient tensor. Those vector can then be used to define the coordinate system transformation matrix $\boldsymbol{Q}$ :

$$
\begin{aligned}
M_{i k} & =F_{i j} M_{j k 0} \\
Q_{1} & =\frac{M_{1}}{\left|M_{1}\right|} \\
Q_{3} & =\frac{M_{1} \times M_{2}}{\left|M_{1} \times M_{2}\right|} \\
Q_{2} & =Q_{3} \times Q_{1}
\end{aligned}
$$

The rotation matrix defined by $\boldsymbol{R}=\boldsymbol{Q}^{T}$ can be used to rotate the various tensor properties listed in Section 5.2 into the rotated spatial reference frame. The rotated unit cell vectors, thermal expansivity tensor and isothermal compressibility tensor are given by:

$$
\begin{aligned}
M_{i k}^{\prime} & =M_{i j} R_{j k} \\
\alpha_{m n}^{\prime} & =R_{m i} \alpha_{i j} R_{j n} \\
S_{\mathrm{T} m n o p}^{\prime} & =R_{m i} R_{n j} S_{\mathrm{T} i j k l} R_{o k} R_{p l}
\end{aligned}
$$


Other anisotropic properties (Section 2) can be calculated using these as a starting point.

5.4 Properties under non-hydrostatic conditions

Elastic properties under mildly non-hydrostatic conditions may be calculated by assuming that the compliance tensor $\mathbb{S}$ is constant at fixed pressure. The Cauchy stress tensor $\boldsymbol{\sigma}$ can be decomposed into hydrostatic and deviatoric parts:

$$
\begin{aligned}
\sigma_{i j} & =s_{i j}+P \delta_{i j} \\
P & =\frac{\sigma_{i i}}{3}
\end{aligned}
$$

As long as the components of the deviatoric stress $s$ are small, the deformation gradient tensor $\boldsymbol{F}$ and molar Gibbs energy $\Delta \mathcal{G}$ at constant temperature can be approximated using $\mathbb{S}$ and $s$ :

$$
\begin{aligned}
F_{i q} & =\left(\frac{x_{i}}{x_{j \mathrm{hyd}}}\right) F_{j q(\mathrm{hyd})} \\
& \sim\left(\delta_{i j}+\varepsilon_{i j}\right) F_{j q(\mathrm{hyd})}=\left(\delta_{i j}-S_{\mathrm{T} i j k l} s_{k l}\right) F_{j q(\mathrm{hyd})} \\
\mathcal{G} & \sim \mathcal{G}_{P}+V_{\text {hyd }} s_{i j} S_{\mathrm{T} i j k l} s_{k l}
\end{aligned}
$$

where the subscript "hyd" indicates the state of the material under hydrostatic conditions, without the deviatoric stress component.

\section{Implementation}

The thermodynamic and thermoelastic properties at any pressure and temperature can now be evaluated. The following steps are involved:

1. Choose a state (pressure and temperature).

2. Compute $f, P_{\mathrm{th}},\left(\partial P_{\mathrm{th}} / \partial f\right) \mid T, \alpha_{V}$ and $\beta_{\mathrm{RT}}$ from the isotropic equation of state of choice.

3. Calculate $\chi_{i j k l}$ (Equation 43), $\left.\left(\partial \chi_{i j k l} / \partial f\right)\right|_{P_{\text {th }}}($ Equation 44$)$ and $\left.\left(\partial \chi_{i j k l} / \partial P_{\text {th }}\right)\right|_{f}$ (Equation 45) from the anisotropic expressions.

4. Calculate the deformation gradient tensor (Equation 51), isothermal compliance tensor (Equations 60) and thermal expansivity tensor (Equations $65)$.

5. For monoclinic and triclinic systems, rotate these tensors into the deformed crystal reference frame (Section 5.3).

6. Calculate other desired anisotropic properties using the (rotated) tensors and Maxwell's relations (Section 2). 


\section{Examples}

\subsection{Cubic symmetry: Periclase $(\mathrm{MgO})$}

Periclase $(\mathrm{MgO})$ is a cubic mineral (space group Fm $\overline{3} \mathrm{~m}$ ) that has been wellstudied for many decades. Here we use weighted least-squares to parameterise an anisotropic equation of state for periclase using isentropic stiffness tensor data from two published studies: Isaak et al (1989) for room pressure data, and Fan et al (2019) for high pressure data. The isotropic equation of state used to provide volume and thermal pressures was that from the dataset of Stixrude and Lithgow-Bertelloni (2011). Fitted parameters are given in Table 2, and the properties are plotted in Figures 3 and 2.

Table 2 Anisotropic parameters used to model periclase. The isotropic equation of state is taken from Stixrude and Lithgow-Bertelloni (2011).

\begin{tabular}{r|rrrrr} 
& $c_{i j 10}$ & $c_{i j 20}$ & $c_{i j 30}$ & $c_{i j 01}$ & $c_{i j 11}$ \\
\hline$c_{11}$ & 0.64435 & 0.97982 & 2.287 & $4.0697 \mathrm{e}-13$ & $8.3313 \mathrm{e}-12$ \\
$c_{12}$ & -0.15551 & -0.48991 & -1.1435 & $-2.0349 \mathrm{e}-13$ & $-4.1657 \mathrm{e}-12$ \\
$c_{44}$ & 1.03 & -3.3939 & -2.0274 & $6.4808 \mathrm{e}-13$ & $5.2939 \mathrm{e}-12$
\end{tabular}

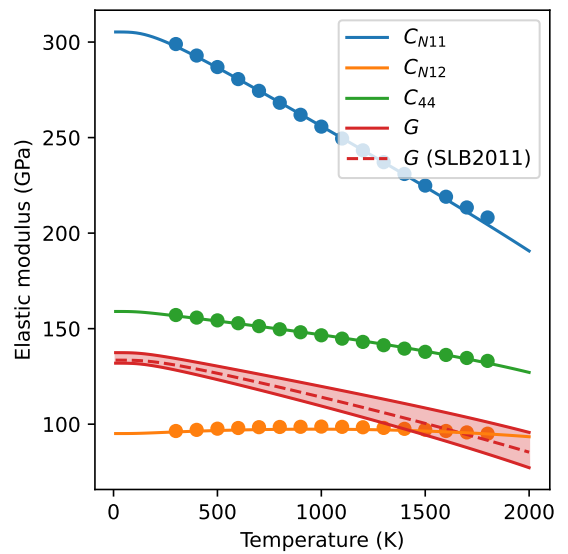

Fig. 2 Elements of the isentropic stiffness tensor for periclase as predicted by the anisotropic model described in the text (Table 2). Properties are computed at atmospheric pressure. Experimental data is from Isaak et al (1989). The filled region spans the range between the Reuss and Voigt bounds on the shear modulus. The dashed line is the prediction for the shear modulus $(G)$ calculated using the Stixrude and Lithgow-Bertelloni (2011) dataset.

Room pressure and room temperature properties are well-retrieved by the anisotropic equation of state. The shear modulus is also well-retrieved under high-pressure conditions, whereas the values of $C_{N 11}$ and $C_{N 12}$ are higher than 

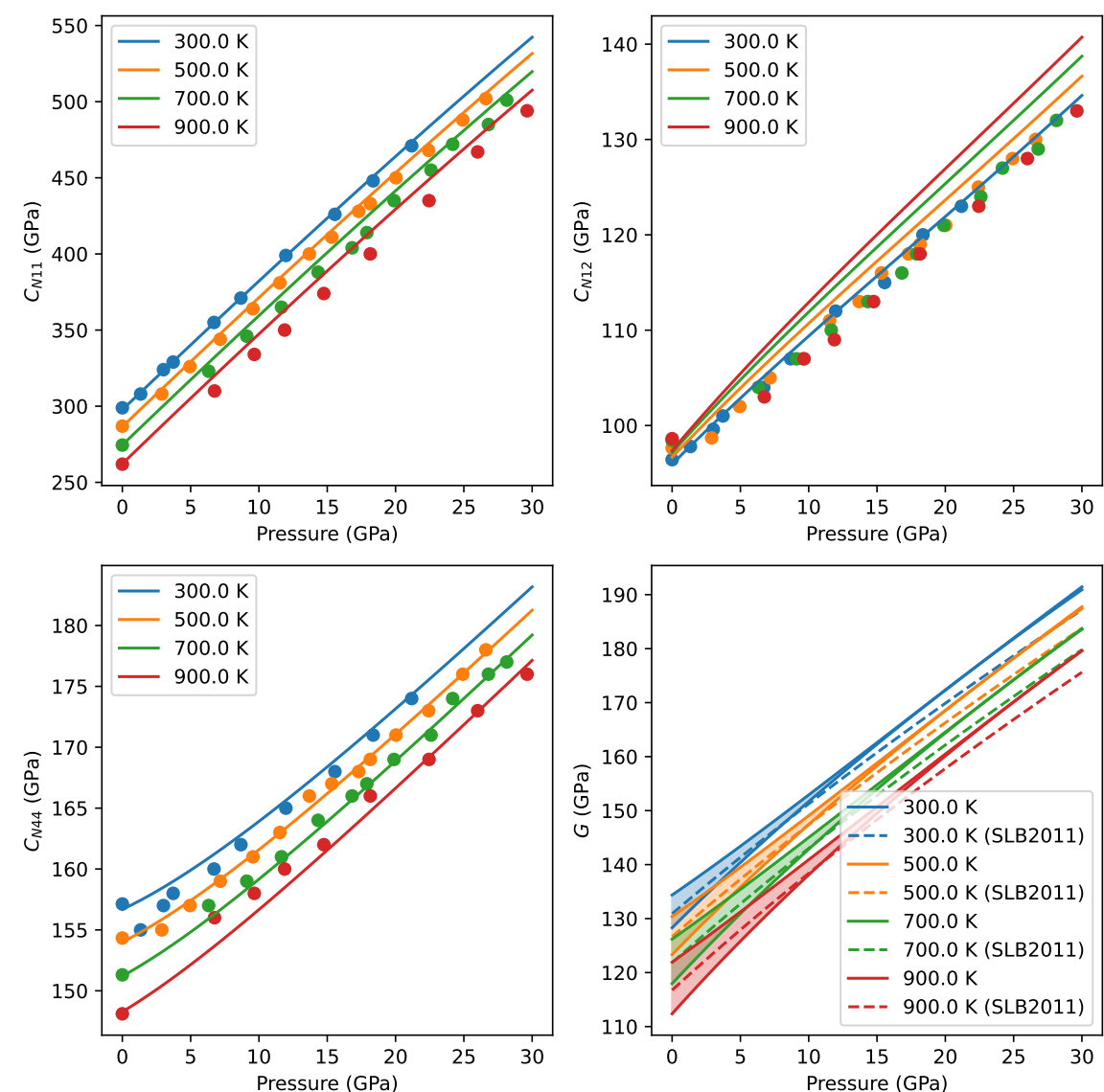

Fig. 3 Elements of the isentropic stiffness tensor for periclase at high pressure as predicted by the anisotropic model described in the text (Table 2). Experimental data at room pressure is from Isaak et al (1989) and data at high pressure is from Fan et al (2019). The filled region in the bottom right hand plot spans the range between the Reuss and Voigt bounds on the shear modulus. Dashed lines are the predictions for the shear modulus $(G)$ calculated using the Stixrude and Lithgow-Bertelloni (2011) dataset.

the experimental values reported by Fan et al (2019). This is a direct consequence of the properties of the isotropic equation of state; the experimental values of $C_{N 11}$ and $C_{N 12}$ are inconsistent with the isentropic compressibility predicted by the Stixrude and Lithgow-Bertelloni (2011) periclase model. 


\subsection{Orthorhombic symmetry: Olivine}

Olivine is an orthorhombic mineral (space group Pbnm). It is the dominant mineral in the Earth's upper mantle, and natural mantle samples have two major endmembers: forsterite $\left(\mathrm{Mg}_{2} \mathrm{SiO}_{4}\right)$ and fayalite $\left(\mathrm{Fe}_{2} \mathrm{SiO}_{4}\right)$. The composition of olivine in the mantle is usually taken to be around $90 \mathrm{~mol} \%$ forsterite, $10 \mathrm{~mol} \%$ fayalite.

One particularly well studied group of olivines are those from the San Carlos Apache Reservation in southeastern Arizona. These olivines have compositions of fog0.4. Their thermal expansion properties have been studied by Singh and Simmons (1976), and their elastic properties by Abramson et al (1997) and Mao et al (2015).

Here, we fit parameters for the anisotropic model using the isentropic stiffness and density data of Mao et al (2015). Rather than use the data of Singh and Simmons (1976) to constrain the thermal expansivity, we instead use the data from Suzuki (1975). Although these data are derived from expansion experiments on an olivine of composition fo92.3 from a lherzolite in Kenya, rather than from San Carlos olivine, the data have significantly lower scatter than the X-ray diffraction data from Singh and Simmons (1976). It is highly unlikely that a less than $2 \%$ change in forsterite content will have a large effect on relative expansion along individual lattice vectors.

We use the Stixrude and Lithgow-Bertelloni (2005) equation of state as the isotropic equation of state. The parameters used in that equation of state are as follows: $V_{0}: 44.10015 \mathrm{~cm}^{3} / \mathrm{mol}, K_{0}: 125.25923 \mathrm{GPa}, K_{0}^{\prime}: 4.28774, \Theta_{0}$ : $790.12334 \mathrm{~K}, \gamma_{0}: 1.11821$, and $q_{0}: 0.66133$. All of these properties apart from $\Theta_{0}$ were optimized during the inversion, along with the anisotropic parameters given in Table 3. The Debye temperature $\Theta_{0}$ was fixed to a molar weighted average of the values for forsterite and fayalite given by Stixrude and LithgowBertelloni (2011).

The parameter value for $V_{0}$ is about $0.25 \%$ larger than the value given by the product of the unit lengths in Abramson et al (1997). The isothermal properties $V_{0}, K_{0}$ and $K_{0}^{\prime}$ are all similar to the values expected from the Stixrude and Lithgow-Bertelloni (2011) dataset. The ambient value of the Grüneisen parameter $\gamma_{0}$ is about $10 \%$ larger than the forsterite and fayalite values given in that dataset. The largest difference is seen in the parameter $q_{0}$, where $q=\partial \ln \gamma / \partial \ln V$. Our best-fit parameter value $(\sim 0.66)$ is significantly smaller than the values for forsterite and fayalite in (Stixrude and Lithgow-Bertelloni, 2011, 2.11 and 3.64 respectively). This parameter is the least well-constrained by the measurements used in this inversion, but values over 1.5 would result in superlinear expansion coefficients for which there is little evidence.

The thermal expansivities for the anisotropic model are given in Figure 4. An excellent fit is obtained for the data from Suzuki (1975).

Isentropic stiffness properties are given in Figure 5, and compared against the experimental data (Mao et al, 2015). The model was able to recreate the pressure dependence of the elastic tensor for all components. The tempera- 
Table 3 Anisotropic parameters used to model olivine. The isotropic equation of state formulation is that of Stixrude and Lithgow-Bertelloni (2005). The parameters used for the volumetric part of the isotropic model are given in the text.

\begin{tabular}{|c|c|c|c|c|c|c|c|c|}
\hline & $c_{i j 10}$ & $c_{i j 20}$ & $c_{i j 30}$ & $c_{i j 01}$ & $c_{i j 11}$ & $c_{i j 21}$ & $c_{i j 31}$ & $c_{i j 02}$ \\
\hline$c_{11}$ & 0.44384 & -0.93819 & 0.85745 & $2.6352 \mathrm{e}-12$ & $3.1099 \mathrm{e}-12$ & $-5.8421 \mathrm{e}-11$ & $1.2221 \mathrm{e}-10$ & $5.1136 \mathrm{e}-23$ \\
\hline$c_{22}$ & 0.77604 & -1.0064 & 5.6678 & $5.124 \mathrm{e}-12$ & $1.5953 \mathrm{e}-11$ & $1.2335 \mathrm{e}-10$ & $-7.6026 \mathrm{e}-11$ & $3.0612 \mathrm{e}-23$ \\
\hline$c_{33}$ & 0.66286 & -0.62954 & 9.071 & $1.705 \mathrm{e}-11$ & $1.9073 \mathrm{e}-11$ & $6.4858 \mathrm{e}-11$ & $2.9973 \mathrm{e}-11$ & $3.6264 \mathrm{e}-23$ \\
\hline$c_{44}$ & 1.9684 & -0.49722 & 20.877 & $-2.6624 \mathrm{e}-11$ & $2.3258 \mathrm{e}-11$ & $-6.2634 \mathrm{e}-11$ & $1.1076 \mathrm{e}-10$ & $-4.995 \mathrm{e}-22$ \\
\hline$c_{55}$ & 1.6114 & -1.8503 & 23.211 & $-3.1569 \mathrm{e}-11$ & $2.6521 \mathrm{e}-11$ & $4.3923 \mathrm{e}-12$ & $4.7107 \mathrm{e}-11$ & $-6.2438 \mathrm{e}-22$ \\
\hline$c_{66}$ & 1.5536 & -1.4269 & 12.645 & $-3.6994 \mathrm{e}-12$ & $5.7178 \mathrm{e}-11$ & $6.4914 \mathrm{e}-11$ & $-3.8195 \mathrm{e}-11$ & $-1.2501 \mathrm{e}-22$ \\
\hline$c_{12}$ & -0.1204 & 0.43893 & -1.1799 & $4.6129 \mathrm{e}-12$ & $-2.214 \mathrm{e}-12$ & $7.8156 \mathrm{e}-11$ & $8.1778 \mathrm{e}-11$ & $-1.3403 \mathrm{e}-24$ \\
\hline$c_{13}$ & -0.10167 & 0.27023 & -2.6814 & $-6.9308 \mathrm{e}-12$ & $-4.0463 \mathrm{e}-12$ & $-3.4918 \mathrm{e}-11$ & $1.0921 \mathrm{e}-10$ & $4.911 \mathrm{e}-24$ \\
\hline$c_{23}$ & -0.21929 & 0.5779 & -3.9368 & $-1.0087 \mathrm{e}-11$ & $-1.2807 \mathrm{e}-11$ & $-1.0813 \mathrm{e}-10$ & $-2.2907 \mathrm{e}-10$ & $-6.2577 \mathrm{e}-23$ \\
\hline
\end{tabular}
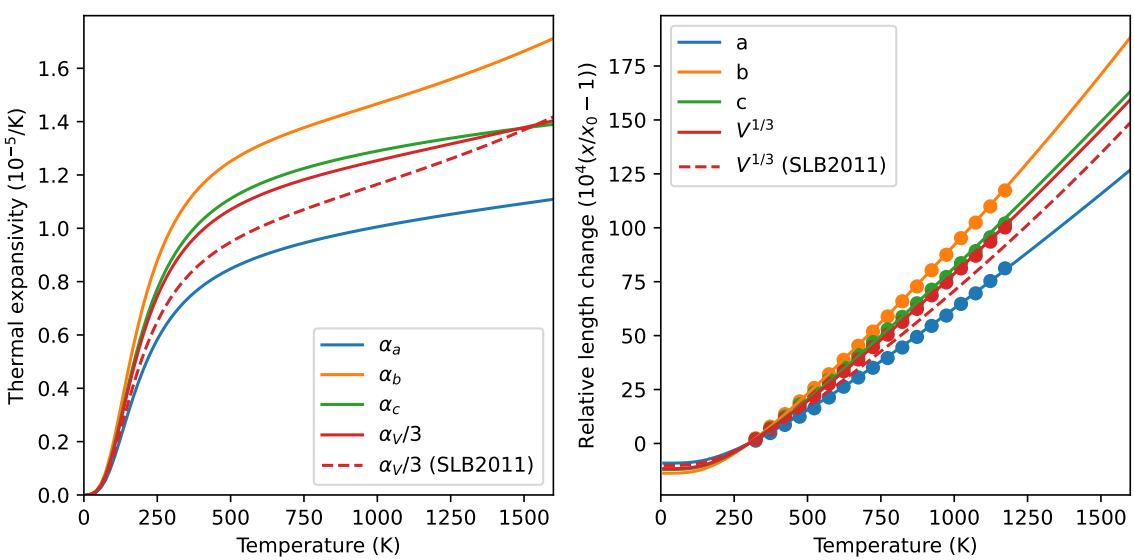

Fig. 4 Thermal expansivity of San Carlos olivine (fo90.4). Experimental data is taken from Suzuki (1975) for an olivine of composition fo92.3, which should exhibit similar relative thermal expansion behaviour. Dashed lines are the properties output from the isotropic model of Stixrude and Lithgow-Bertelloni (2011).

ture dependence of the $C_{\mathrm{N} i i}$ components was also well retrieved. The model temperature-dependence of the off-diagonal components $C_{\mathrm{N} 12}, C_{\mathrm{N} 13}$ and $C_{\mathrm{N} 23}$ was smaller than the observed change. This is unlikely to be a shortcoming of the anisotropic model, but rather reflects that there is some incompatibility in the experimental constraints.

The anisotropic equation of state can be used to calculate any elastic properties at any given pressure and temperature. Examples are given for San Carlos olivine at $3 \mathrm{GPa}$ and $1600 \mathrm{~K}$, conditions similar to those at the base of oceanic lithosphere (McKenzie et al, 2005). 

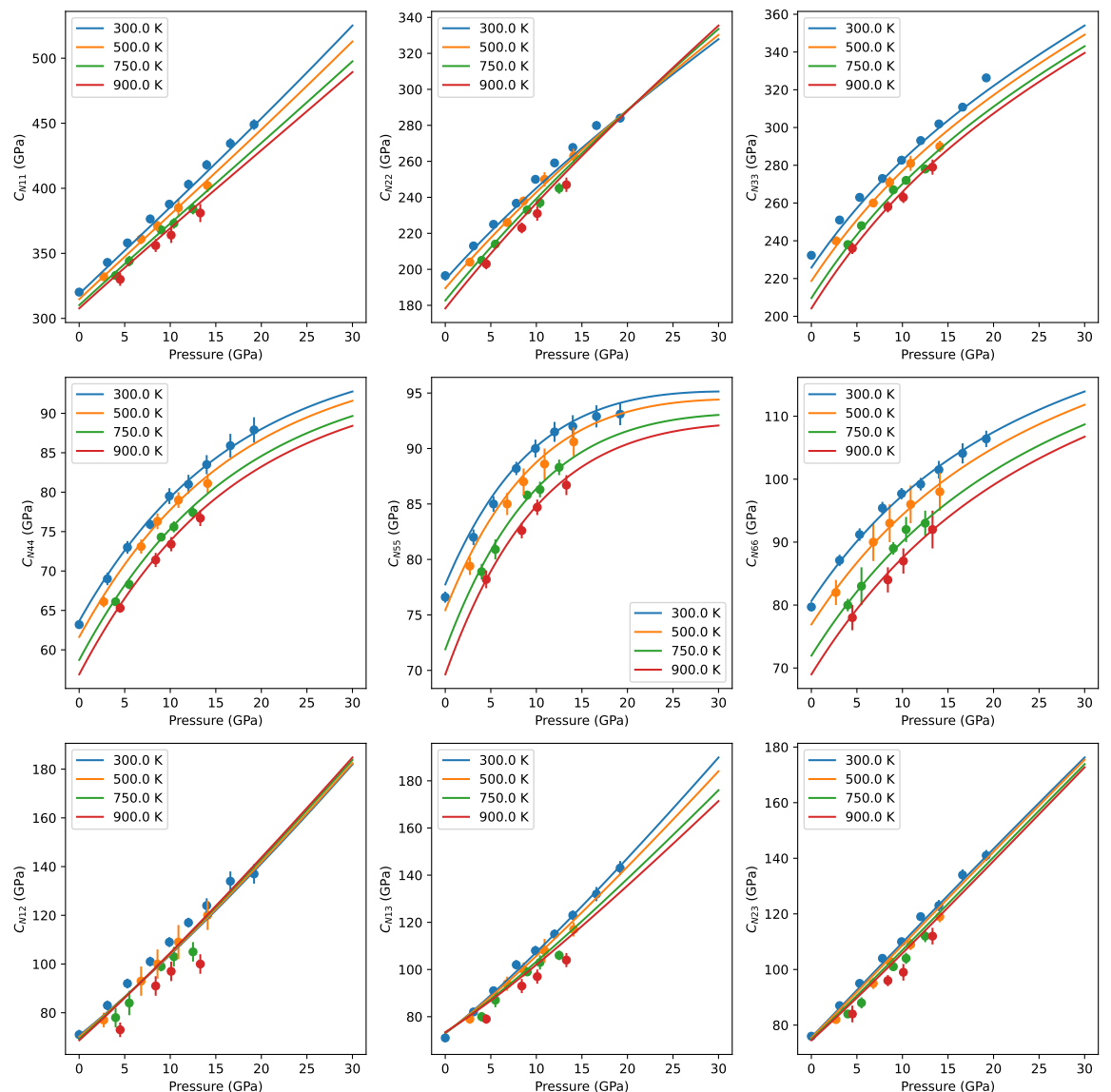

Fig. 5 Elements of the isentropic stiffness tensor for San Carlos olivine (fog0.4) as predicted by the anisotropic model described in the text (Table 3). High pressure data is from Mao et al (2015). 

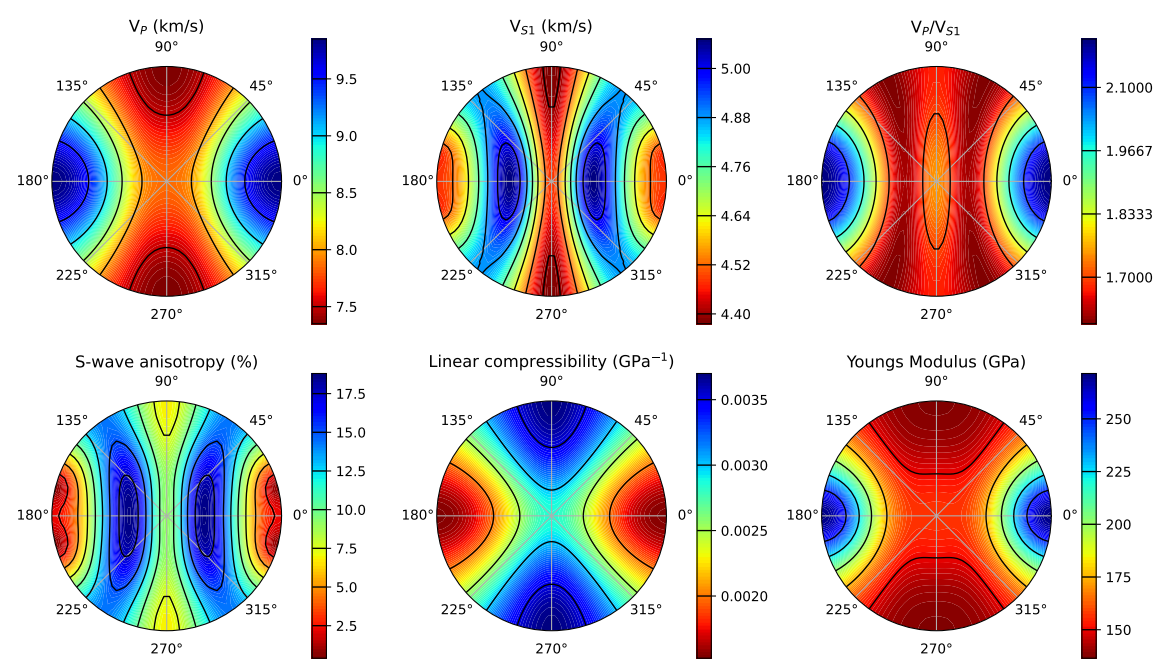

Fig. 6 Seismic properties for San Carlos olivine (fo90.4) at 3 GPa and $1600 \mathrm{~K}$ as predicted by the anisotropic model described in the text (Table 3). All properties are given for conditions of constant entropy (as required for the calculation of seismic properties). 


\section{Discussion}

8.1 Consistent inversions of experimental data

Single crystal experimental data is often provided in the form of volumes, lattice parameters (Equation 69) and components of the isentropic compliance tensor as a function of pressure and temperature (Angel et al, 2009). In the absence of an equation of state that can convert between isentropic and isothermal compliances, it has been tempting to take the easy route and assume that the two are equal, which is only true at zero Kelvin. Although the difference is small (on the order of $1 \%$ ) at room temperature, it increases with increasing temperature, posing problems for fitting data to thermal equations of state.

The equation of state presented here allows all elastic data to be fit simultaneously and consistently. This will facilitate consistent joint inversions of data from different analytical techniques, such as X-ray diffraction and Brillouin spectroscopy (Kurnosov et al, 2017; Criniti et al, 2021; Siersch et al, 2021).

\subsection{Elastic stability}

The anisotropic formulation described in Section 4 does not guarantee that the tensor is elastically stable. A material is elastically stable when the stiffness/compliance tensor is positive definite (Born, 1940; Born and Huang, 1954), as any change in strain increases the internal energy. This positive definite-ness can be expressed as a series of inequalities composed of a elements of the stiffness tensor (Mouhat and Coudert, 2014) or compliance tensor.

If anisotropic equations of state are derived from experimental data, that data should indicate that the material is elastically stable. However, users should also ensure that the parameterised functions for $\mathbb{S}$ are indeed positive definite under the conditions of interest.

Elastic stability does not imply anything about thermodynamic stability; an elastically stable mineral at a given pressure and temperature can still be thermodynamically metastable relative to another assemblage of the same composition.

\subsection{Code availability}

The anisotropic equation of state described in this paper is provided as a contribution to the BurnMan open source software project (currently available on a branch: https://github.com/bobmyhill/burnman/tree/anisotropic_eos) (Cottaar et al, 2014; Myhill et al, 2021). 


\section{Acknowledgements}

This paper was born thanks to discussions in Bristol with James Wookey and Mike Kendall, and in Utrecht with Cedric Thieulot. It was revived from a moribund state thanks to discussions with Jean-Paul Montagner at the CLEEDI workshop held in Foix in August 2021. This work was supported by NERC Large Grant MC-squared (Award No. NE/T012633/1). Any mistakes or oversights are of course my own.

\section{A Derivation of the thermodynamic anisotropic Grüneisen tensor}

There are various non-equivalent forms for the Grüneisen tensor in the literature (e.g. Key, 1967; Barron and Munn, 1967; Cantrell, 1980; Haussühl, 2003). The differences appear to have arisen from various choices in the definition of the tensor and varied approaches to subscripting isentropic, isothermal and isenergetic forms of the stiffness tensor. The form adopted in Equation 18 has the same form as the second expression given by Barron and Munn (1967).

The isotropic Grüneisen parameter can be defined as:

$$
\gamma=-\left.\frac{\partial \ln T}{\partial \ln V}\right|_{N}=-\frac{1}{T} \frac{\partial^{2} E}{\partial \ln V \partial N}
$$

This equation can be generalised to anisotropic materials:

$$
\gamma T=-\frac{\partial^{2} E}{\partial \varepsilon \partial N}
$$

Now, to derive Equation 18, we first write out the total differential of $\boldsymbol{\sigma}(\varepsilon, N)$ :

$$
d \boldsymbol{\sigma}=\left.\frac{\partial \boldsymbol{\sigma}}{\partial \varepsilon}\right|_{N} d \varepsilon+\left.\frac{\partial \boldsymbol{\sigma}}{\partial N}\right|_{\partial \varepsilon} d N
$$

We can also express the infinitesimal strain tensor and entropy as functions of stress and temperature. Expanding the above expression using the chain rule gives:

$$
d \boldsymbol{\sigma}=\left.\frac{\partial \boldsymbol{\sigma}}{\partial \boldsymbol{\varepsilon}}\right|_{N}\left(\left.\frac{\partial \varepsilon}{\partial \boldsymbol{\sigma}}\right|_{T} d \boldsymbol{\sigma}+\left.\frac{\partial \varepsilon}{\partial T}\right|_{\boldsymbol{\sigma}} d T\right)+\left.\frac{\partial \boldsymbol{\sigma}}{\partial N}\right|_{\boldsymbol{\varepsilon}}\left(\left.\frac{\partial N}{\partial \boldsymbol{\sigma}}\right|_{T} d \boldsymbol{\sigma}+\left.\frac{\partial N}{\partial T}\right|_{\boldsymbol{\sigma}} d T\right)
$$

Replacing terms with their thermodynamic equivalents, we have:

$$
d \boldsymbol{\sigma}=\mathbb{C}_{\mathrm{N}}\left(\mathbb{S}_{T} d \boldsymbol{\sigma}-\boldsymbol{\alpha} d T\right)+\frac{\gamma T}{V}\left(-\boldsymbol{\alpha} V d \boldsymbol{\sigma}+\frac{C_{\boldsymbol{\sigma}}}{T} d T\right)
$$

Taking the derivative with respect to temperature at constant stress yields:

$$
\begin{aligned}
0 & =\mathbb{C}_{\mathrm{N}}(-\boldsymbol{\alpha})+\frac{\gamma T}{V}\left(\frac{C_{\boldsymbol{\sigma}}}{T}\right) \\
\gamma & =\mathbb{C}_{\mathrm{N}} \boldsymbol{\alpha} \frac{V}{C_{\boldsymbol{\sigma}}}
\end{aligned}
$$

which is the form presented in Equation 18.

For isotropic materials, the Grüneisen tensor will be a diagonal matrix with each diagonal element equal to the scalar Grüneisen parameter. 


\section{B Alternative descriptions of the thermal expansivity and isothermal compressibility}

Starting from Equation 51, we have:

$$
\left(\ln _{\mathrm{M}} F\right)_{i j}=\chi_{i j k l} \delta_{k l}
$$

Taking the total derivative of this tensor with respect to $P$ and $T$ (Section 5.2):

$$
d\left(\ln _{\mathrm{M}} F\right)_{i j}=g_{i j k l} \delta_{k l} d P+h_{i j k l} \delta_{k l} d T
$$

Recognising that $g_{i j k l}=-S_{\mathrm{T} i j k l}$ and $h_{i j k l} \delta_{k l}=\alpha_{i j}$ (also Section 5.2), we can define alternative expressions for $\alpha_{i j}$ and $\beta_{\mathrm{T} i j}$, the isothermal compressibility:

$$
\begin{gathered}
\alpha_{i j}=\left.\frac{\partial\left(\ln _{\mathrm{M}} \boldsymbol{F}\right)_{i j}}{\partial T}\right|_{P} \\
\beta_{\mathrm{T} i j}=S_{i j k l} \delta_{k l}=-\left.\frac{\partial\left(\ln _{\mathrm{M}} \boldsymbol{F}\right)_{i j}}{\partial P}\right|_{T}
\end{gathered}
$$

As described in Section 5.3, for monoclinic and triclinic materials, these tensors must be rotated into the material reference frame before analysis.

\section{References}

Abramson EH, Brown JM, Slutsky LJ, Zaug J (1997) The elastic constants of San Carlos olivine to 17 GPa. Journal of Geophysical Research 102(B6):12,253-12,263, DOI 10.1029/ $97 \mathrm{jb00682}$

Anderson OL (1968) On the use of ultrasonic and shock-wave data to estimate compressions at extremely high pressures. Physics of the Earth and Planetary Interiors 1(3):169-176, DOI 10.1016/0031-9201(68)90006-x

Angel RJ, Jackson JM, Reichmann HJ, Speziale S (2009) Elasticity measurements on minerals: a review. European Journal of Mineralogy 21:525-550, DOI 10.1127/0935-1221/ 2009/0021-1925

Barron THK, Munn RW (1967) Analysis of the thermal expansion of anisotropic solids: Application to zinc. Philosophical Magazine 15(133):85-103, DOI 10.1080/ 14786436708230352

Birch F (1947) Finite Elastic Strain of Cubic Crystals. Physical Review 71:809-824, DOI 10.1103/PhysRev.71.809

Born M (1940) On the stability of crystal lattices. i. Mathematical Proceedings of the Cambridge Philosophical Society 36(2):160-172, DOI 10.1017/S0305004100017138

Born M, Huang K (1954) Dynamical theory of crystal lattices. Clarendon press

Cantrell JH (1980) Generalized grüneisen tensor from solid nonlinearity parameters. Physical Review B 21(10):4191-4195, DOI 10.1103/physrevb.21.4191

Cottaar S, Heister T, Rose I, Unterborn C (2014) Burnman: A lower mantle mineral physics toolkit. Geochemistry, Geophysics, Geosystems 15(4):1164-1179, DOI https://doi.org/10.1002/2013GC005122, URL https://agupubs.onlinelibrary.wiley . com/doi/abs/10.1002/2013GC005122, https://agupubs.onlinelibrary.wiley.com/doi/ pdf/10.1002/2013GC005122

Criniti G, Kurnosov A, Boffa Ballaran T, Frost DJ (2021) Single-Crystal Elasticity of MgSiO 3 Bridgmanite to Mid-Lower Mantle Pressure. Journal of Geophysical Research: Solid Earth 126(5), DOI 10.1029/2020jb020967

Davies GF (1974) Effective elastic moduli under hydrostatic stress - I. quasi-harmonic theory. Journal of Physics and Chemistry of Solids 35:1513-1520, DOI 10.1016/ S0022-3697(74)80279-9 
de Jong M, Chen W, Angsten T, Jain A, Notestine R, Gamst A, Sluiter M, Krishna Ande C, van der Zwaag S, Plata JJ, Toher C, Curtarolo S, Ceder G, Persson KA, Asta M (2015) Charting the complete elastic properties of inorganic crystalline compounds. Scientific Data, Volume 2, id 150009 (2015) 2:150009, DOI 10.1038/sdata.2015.9

Fan D, Fu S, Yang J, Tkachev SN, Prakapenka VB, Lin JF (2019) Elasticity of single-crystal periclase at high pressure and temperature: The effect of iron on the elasticity and seismic parameters of ferropericlase in the lower mantle. American Mineralogist 104(2):262-275, DOI 10.2138/am-2019-6656, URL https://doi.org/ 10.2138/am-2019-6656, https://pubs.geoscienceworld.org/ammin/article-pdf/104/ 2/262/4627943/am-2019-6656.pdf

Haussühl S (2003) XXIII - Microscopic and macroscopic properties of solids. In: Leiber CO, Dobratz B (eds) Assessment of Safety and Risk with a Microscopic Model of Detonation, Elsevier Science, Amsterdam, pp 493-554, DOI https://doi.org/10.1016/ B978-044451332-8/50024-0, URL https://www.sciencedirect.com/science/article/ pii/B9780444513328500240

Holland TJB, Powell R (2011) An improved and extended internally consistent thermodynamic dataset for phases of petrological interest, involving a new equation of state for solids. Journal of Metamorphic Geology 29:333-383, DOI 10.1111/j.1525-1314.2010. 00923.x

Holland TJB, Hudson NFC, Powell R, Harte B (2013) New Thermodynamic Models and Calculated Phase Equilibria in NCFMAS for Basic and Ultrabasic Compositions through the Transition Zone into the Uppermost Lower Mantle. Journal of Petrology 54:19011920, DOI 10.1093/petrology/egt035

Isaak DG, Anderson OL, Goto T (1989) Measured elastic moduli of single-crystal mgo up to $1800 \mathrm{k}$. Physics and Chemistry of Minerals 16(7):704-713, DOI 10.1007/BF00223321, URL https://doi.org/10.1007/BF00223321

Ita J, Stixrude L (1992) Petrology, elasticity, and composition of the mantle transition zone. Journal of Geophysical Research 97:6849-6866, DOI 10.1029/92JB00068

Keane A (1954) An investigation of finite strain in an isotropic material subjected to hydrostatic pressure and its seismological applications. Australian Journal of Physics 7(2):322, DOI $10.1071 /$ ph540322

Key SW (1967) Grüneisen tensor for anisotropic materials. Journal of Applied Physics 38(7):2923-2928, DOI 10.1063/1.1710025

Kurnosov A, Marquardt H, Frost DJ, Ballaran TB, Ziberna L (2017) Evidence for a Fe ${ }^{3+}$. rich pyrolitic lower mantle from (Al,Fe)-bearing bridgmanite elasticity data. Nature 543:543-546, DOI 10.1038/nature21390

Mainprice D, Hielscher R, Schaeben H (2011) Calculating anisotropic physical properties from texture data using the mtex open-source package. Geological Society, London, Special Publications 360(1):175-192

Mao Z, Fan D, Lin JF, Yang J, Tkachev SN, Zhuravlev K, Prakapenka VB (2015) Elasticity of single-crystal olivine at high pressures and temperatures. Earth and Planetary Science Letters 426:204-215, DOI https://doi.org/10.1016/j.epsl.2015.06.045, URL https://www . sciencedirect.com/science/article/pii/S0012821X15004082

Marquardt H, Thomson AR (2020) Experimental elasticity of Earth's deep mantle. Nature Reviews Earth and Environment 1(9):455-469, DOI 10.1038/s43017-020-0077-3

McKenzie D, Jackson J, Priestley K (2005) Thermal structure of oceanic and continental lithosphere. Earth and Planetary Science Letters 233(3-4):337-349, DOI 10.1016/j.epsl. 2005.02.005

Mouhat F, Coudert FX (2014) Necessary and sufficient elastic stability conditions in various crystal systems. Phys Rev B 90:224,104, DOI 10.1103/PhysRevB.90.224104, URL https: //link.aps.org/doi/10.1103/PhysRevB.90.224104

Murnaghan FD (1937) Finite deformations of an elastic solid. American Journal of Mathematics 59(2):235-260, DOI 10.2307/2371405, URL http://www.jstor.org/stable/ 2371405

Myhill R, Cottaar S, Heister T, Rose I, Unterborn C (2021) Burnman v0.10.0. DOI 10.5281/ zenodo.5155442, URL https://doi.org/10.5281/zenodo. 5155442

Nye JF, et al (1985) Physical properties of crystals: their representation by tensors and matrices. Oxford university press 
Poirier JP, Tarantola A (1998) A logarithmic equation of state. Physics of the Earth and Planetary Interiors 109(1-2):1-8, DOI 10.1016/s0031-9201(98)00112-5

Reiner M (1958) Rheology. In: Flügge S (ed) Elasticity and Plasticity / Elastizität und Plastizität, Springer, Berlin, Heidelberg, Berlin, Heidelberg, pp 434-550, DOI https:// doi.org/10.1007/978-3-662-43081-1_4

Siersch NC, Kurnosov A, Criniti G, Ishii T, Boffa Ballaran T, Frost DJ (2021) The elastic properties and anisotropic behavior of $\mathrm{MgSiO}_{3}$ akimotoite at transition zone pressures. Physics of the Earth and Planetary Interiors 320:106,786, DOI https://doi.org/10. 1016/j.pepi.2021.106786, URL https://www.sciencedirect.com/science/article/pii/ S0031920121001448

Singh HP, Simmons G (1976) X-ray determination of thermal expansion of olivines. Acta Crystallographica Section A 32(5):771-773, DOI https://doi.org/ 10.1107/S0567739476001575, URL https://onlinelibrary.wiley.com/doi/abs/10. 1107/S0567739476001575, https://onlinelibrary.wiley.com/doi/pdf/10.1107/ S0567739476001575

Stacey F, Davis P (2004) High pressure equations of state with applications to the lower mantle and core. Physics of the Earth and Planetary Interiors 142(3-4):137-184, DOI 10.1016/j.pepi.2004.02.003

Stacey FD, Brennan BJ, Irvine RD (1981) Finite strain theories and comparisons with seismological data. Geophysical Surveys 4(3):189-232, DOI 10.1007/bf01449185

Stackhouse S, Brodholt JP (2007) The high-temperature elasticity of $\mathrm{MgSiO}_{3}$ postperovskite. Washington DC American Geophysical Union Geophysical Monograph Series 174:99-113

Stixrude L, Lithgow-Bertelloni C (2005) Thermodynamics of mantle minerals - I. Physical properties. Geophysical Journal International 162:610-632, DOI 10.1111/j.1365-246X. 2005.02642.x

Stixrude L, Lithgow-Bertelloni C (2011) Thermodynamics of mantle minerals - II. Phase equilibria. Geophysical Journal International 184:1180-1213, DOI 10.1111/j.1365-246X 2010.04890.x

Suzuki I (1975) Thermal expansion of periclase and olivine, and their anharmonic properties. Journal of Physics of the Earth 23(2):145-159, DOI 10.4294/jpe1952.23.145

Thomsen L (1972) The fourth-order anharmonic theory: Elasticity and stability. Journal of Physics and Chemistry of Solids 33(2):363 - 378, DOI 10.1016/0022-3697(72)90018-2, URL http://www.sciencedirect.com/science/article/pii/0022369772900182

Thurston RN (1967) Calculation of lattice-parameter changes with hydrostatic pressure from third-order elastic constants. The Journal of the Acoustical Society of America 41(4B):1093-1111, DOI 10.1121/1.1910442

Truesdell C (1952) The mechanical foundations of elasticity and fluid dynamics. Journal of Rational Mechanics and Analysis 1:125-300, URL http://www.jstor.org/stable/ 24900260

Vinet P, Ferrante J, Rose JH, Smith JR (1987) Compressibility of solids. Journal of Geophysical Research 92(B9):9319, DOI 10.1029/jb092ib09p09319

Voigt W (1928) Lehrbuch der kristallphysik:(mit ausschluss der kristalloptik), vol 34. BG Teubner 